\title{
Influence of regular exercise training on post-exercise hemodynamic regulation to orthostatic challenge
}

\author{
Jun Sugawara ${ }^{1}$, Hidehiko Komine ${ }^{1}$, Taiki Miyazawa ${ }^{1,2}$, Tomoko Imai ${ }^{3}$ and Shigehiko Ogoh ${ }^{2}$ \\ ${ }^{1}$ Human Technology Research Institute, National Institute of Advanced Industrial Science and Technology, Ibaraki, Japan \\ ${ }^{2}$ Department of Biomedical Engineering, Toyo University, Kawagoe, Japan \\ ${ }^{3}$ Graduate School of Comprehensive Human Sciences, University of Tsukuba, Tsukuba, Japan
}

Edited by:

Qi Fu, UT Southwestern Medical

Center, USA

Reviewed by:

Stuart Best, James Cook University,

Australia

Megan Wenner, University of

Delaware, USA

${ }^{*}$ Correspondence:

Jun Sugawara, Human Technology

Research Institute, National Institute

of Advanced Industrial Science and

Technology, 1-1-1 Higashi, Tsukuba,

Ibaraki 305-8566, Japan

e-mail: jun.sugawara@aist.go.jp
To prevent orthostatic hypotension, arterial blood pressure (BP) is neurally and hormonally regulated via increases in heart rate (HR) and peripheral vascular tone. After dynamic exercise, however, the latter arm is blunted because of the increased vasodilators in exercised muscles. Orthostatic tachycardia is likely a more important compensatory mechanism for post-exercise orthostatic intolerance in individuals who have higher leg vasodilator capacity, such as endurance-trained athletes. To test the hypothesis that regular endurance training was associated with the greater augmentation of tachycardia response to post-exercise orthostasis, we compared hemodynamic responses to 5 -min $60^{\circ}$ head-up tilt (HUT) before and after $60 \mathrm{~min}$ of cycling at $70 \%$ of HR reserve in the endurance-trained $(n=8)$ and sedentary men $(n=9)$. Calf peak vascular conductance was $62 \%$ greater in the endurance-trained than the sedentary $(P<0.001)$. After the exercise, the HUT-induced reduction of SV was significantly augmented in the endurance-trained (from $-27.7 \pm 6.9$ to $-33.7 \pm 7.7 \mathrm{ml}, P=0.03$ ) but not in their sedentary peers. Nevertheless, MAP was well maintained during post-exercise HUT even in the endurance-trained (from $81 \pm 10$ to $80 \pm 8 \mathrm{mmHg}$ ). Tachycardia responses during sustained orthostasis were significantly increased in the sedentary (1.3-fold vs. pre-exercise) and more in the endurance-trained (2.0-fold). The augmented response of HUT-induced tachycardia was greater in the endurance-trained than the sedentary $(P=0.04)$. Additionally, cardiovagal baroreflex sensitivity (BRS), evaluated by the HR response to the hypotensive perturbation, was improved after the exercise in the endurance-trained (from $-0.56 \pm 0.32$ to $-1.03 \pm 0.26 \mathrm{bpm} / \mathrm{mmHg}, P=0.007$ ) but not in the sedentary. These results suggest that in the endurance-trained men the increased orthostatic tachycardia and augmented cardiovagal BRS may favorably mitigate accumulated risks for orthostatic intolerance in the early phase of post-exercise.

Keywords: exercise training, orthostatic tolerance, baroreflex, tachycardia, stroke volume

\section{INTRODUCTION}

Orthostasis, a frequent disturbance of hemodynamic condition in humans, evokes short-term central hypovolemia, which results in an abrupt drop of arterial blood pressure (BP). In such a condition, normally, BP is well regulated as the unloading of the arterial and cardiopulmonary baroreceptors compensates for the decrease in stroke volume (SV) by evoking neurally and hormonally mediated increases in heart rate (HR) and peripheral vascular resistance (Victor and Leimbach, 1987; Ogoh et al., 2003; Fu et al., 2004). However, in well-trained endurance athletes, BP is not well regulated to orthostatic stress, which leads to orthostatic hypotension and syncope. This is partly due to higher leg maximal vasodilator capacity and greater decline in SV during orthostatic stimulation (Levine et al., 1991).

It is well-known that a prolonged bout of endurance exercise is associated with orthostatic intolerance (Lucas et al., 2008; Murrell et al., 2011; Willie et al., 2013). On the other hand, following a single bout of dynamic exercise even at moderate intensity and duration, local and systemic vasodilators (i.e., nitric oxide, prostacycline, endothelium-derived hyperpolarizing factor, adenosine, histamine) (Bhagyalakshmi and Frangos, 1989; Nagao and Vanhoutte, 1993; Dinenno and Joyner, 2004; Emhoff et al., 2011; Joyner and Casey, 2014); are elicited and there is blunted vasoconstriction in exercised muscles (Halliwill et al., 1996a). This results in the reduced venous return and augments the decline in SV during orthostasis, a risk for orthostatic hypotension and intolerance. In this context, orthostatic tachycardia is likely a more important compensatory mechanism for postexercise orthostatic hypotension in individuals who have higher leg vasodilator capacity (i.e., endurance-trained athletes).

With this information as background, we hypothesized that regular endurance training was associated with the greater augmentation of the tachycardia response to post-exercise orthostatic stimulation. To test this hypothesis, we compared hemodynamic responses to 5-min orthostatic stimulation before and after 1-h moderate intensity cycling between the endurance-trained and 
the sedentary men. Additionally, changes in autonomic function, such as steady-state heart rate variability (HRV) and cardiovagal baroreflex sensitivity (BRS), were also evaluated in these individuals.

\section{MATERIALS AND METHODS SUBJECTS}

A total of 17 apparently healthy men (nine sedentary and eight endurance-trained) participated. The habitual physical activity status of each subject was screened prior to the experiment. Endurance-trained men were long-distance runners or triathletes and trained more than 5 days/week regularly (more than 1 year). Sedentary men have not engaged in exercise training regularly (less than once/week) for at least the last year. All subjects had no apparent cardiovascular disease as assessed by medical history. Subjects who were current smokers or smoked within the past 2 years were excluded. This study was reviewed and approved by the Institutional Review Board in the National Institute of Advanced Industrial Science and Technology (\#2010-252). Additionally, all procedures conform to the ethical guidelines of Helsinki Declaration. All potential risks and procedures of the study were explained to the subjects, and they gave their written informed consent to participate in the study.

\section{EXPERIMENTAL PROTOCOL}

The subjects abstained from alcohol, caffeine, and vigorous exercise for at least $24 \mathrm{~h}$ before the experiments. All measurements were conducted after a $3 \mathrm{~h}$ fast in a quiet, temperature-controlled room $\left(\approx 26 \mathrm{C}^{\circ}\right)$. The timeline of the experiment was shown in Figure 1. At first, height and body weight were measured. Then, after at least $10 \mathrm{~min}$ of quiet rest, each subject underwent cardiac echo measurement, which was followed by the head-up tilt (HUT) test. The HUT test consisted of $5 \mathrm{~min}$ of supine resting and 5 min of $60^{\circ}$ HUT. During HUT, subjects stood on the foot rest of the tilt bed and were instructed not to move or contract muscles in their lower limbs voluntarily. Hemodynamic variables were continuously monitored throughout the HUT test.

After all pre-exercise measurements, each subject underwent 60 min of cycling exercise. For prevention of dehydration, subjects were given a bottle containing $200 \mathrm{ml}$ of water and instructed to

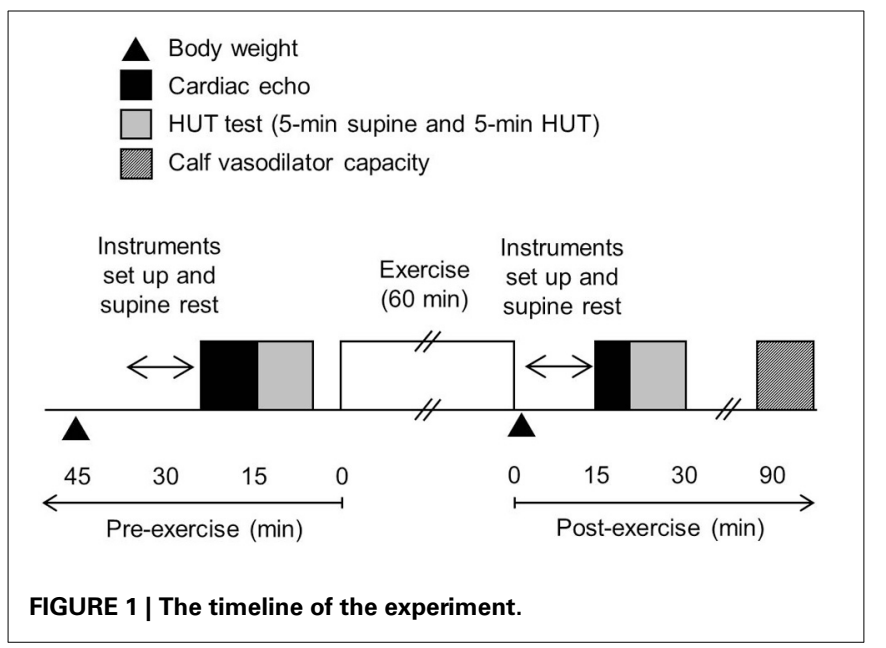

drink it during exercise. Immediately after exercise, each subject's body weight was re-measured. To control the influence of water intake, each subject ingested water immediately after the exercise as the total amount of water was equivalent to $30 \%$ of individual actual body weight loss (e.g., \{[pre-exercise body weight] [post-exercise body weight] - [weight of water ingested during exercise] $\} \times 0.3$ ).

The post-exercise cardiac echo measurement, HUT test, and leg vasodilator capacity measurement were performed 15, 20, and $90 \mathrm{~min}$ after the termination of exercise, respectively.

\section{EXERCISE BOUT}

Using an electrically-braked cycle ergometer (232CXL; COMBI WELLNESS, Tokyo, Japan), each subject underwent a cycling exercise bout consisting of $10 \mathrm{~min}$ warm-up and $50 \mathrm{~min}$ main exercise corresponding to work rates at 65 and $75 \%$ of $\mathrm{HR}$ reserve, respectively. $\mathrm{HR}$ reserve was calculated from the baseline (up-right resting) $\mathrm{HR}$ and age-predicted maximal HR (e.g., 220-age). During the exercise bout, HR was monitored continuously. An investigator supervised each subject to perform cycling exercise around the target intensities. When HR dissociated from the target (more than $5 \mathrm{bpm}$ ), the exercise intensity was modulated appropriately.

\section{MEASUREMENTS \\ Body weight}

Upon arrival to the laboratory, subjects voided their bladder and recorded their nude body weight (via digital weight scale with $20 \mathrm{~g}$ of weight increment, BWB-200, TANITA, Tokyo, Japan). Following the exercise bout, subjects dried themselves with a towel and measured nude body weight again. Body weight loss was reported as the combined amount of the difference between pre- and post-body weight and the amount of ingested water. Body mass index (BMI) was calculated with pre-exercise body weight and height.

\section{LV property}

Echocardiography was used to measure LV end-diastolic diameter (LVEDD), LV wall thickness (LVWT), LV mass (LVM), and fractional shortening (FS) according to established guidelines (Cheitlin et al., 2003).

$$
\begin{aligned}
\operatorname{LVWT}(\mathrm{mm})= & (\mathrm{PWT}+\mathrm{IVST}) / 2 \\
\operatorname{LVM}(\mathrm{g})= & 1.04 \times\left[(\mathrm{LVEDD}+\mathrm{PWT}+\mathrm{IVST})^{3}-(\mathrm{LVEDD})^{3}\right] \\
& -14(\text { Devereux and Reichek, } 1977) \\
\mathrm{FS}(\%)= & (\text { LVEDD }- \text { LVESD }) / \mathrm{LVEDD}^{*} 100
\end{aligned}
$$

where PWT is posterior wall thickness, IVST is interventricular septal thickness, and LVESD is LV end-systolic internal diameter. LVEDD and LVM were normalized by the body surface area and LVWT was normalized by LVEDD. These were reported as LVEDD index, LVM index, and LVWT index, respectively. 


\section{Hemodynamic variables}

HR was recorded with ECG (ML132 Bio Amp, ADInstruments, Colorado Springs, CO). Using a non-invasive beat-to-beat BP monitoring system (Finometer, TNO TPD Biomedical Instruments, Amsterdam, Netherlands), finger arterial pressure wave forms were continuously recorded using the right index finger fixed at the heart level, and calibrated by oscillometric $\mathrm{BP}$ measured in the right arm. By use of software for beat-to-beat analysis of hemodynamic variables (BeatScope 1.1, TNO TPD Biomedical Instruments, Amsterdam, Netherlands), brachial arterial pressure was estimated by a filtering of the finger arterial waveforms. Beat-to-beat SV was computed with the Modelflow method, incorporating age, sex, height, and weight (Wesseling et al., 1993; Sugawara et al., 2003), and then calibrated by the Doppler-echocardiography method: Modelflow SV was made equal to the simultaneously measured SV by Dopplerechocardiography for each subject. Doppler-echo and Modelflow SV measurements were performed prior to the pre- and postexercise HUT tests in a lateral recumbent position. Cardiac output $(\mathrm{CO})$ was calculated as $\mathrm{SV} \times \mathrm{HR}$. Total peripheral resistance (TPR) was calculated as MAP/CO. These parameters were normalized by body surface area (e.g., SV index, CO index, and TPR index, respectively). Continuous data for the last $1 \mathrm{~min}$ during each posture were averaged and reported.

\section{HRV and BRS}

Steady-state cardiovagal outflow was evaluated by power spectral analysis of the beat-to-beat variability of R-R interval (Task Force of the European Society of Cardiology and the North American Society of Pacing and Electrophysiology, 1996). The FFT analyses were performed on segments of 128 consecutive beats during the latter phase of each posture. High frequency (HF: $0.15-0.40 \mathrm{~Hz}$ ) power normalized by total power was defined as an index of vagal activity.

Cardiovagal BRS was quantified with the reduction in MAP (from supine MAP to the nadir MAP) and the followed increase in HR (from supine HR to the peak HR) observed an instant headup stimulation (e.g., $\Delta \mathrm{HR} / \Delta \mathrm{MAP}$ ) (Smith and Raven, 1986). The time required to $60^{\circ}$ head up was $1-2 \mathrm{~s}$ in all subjects.

\section{Leg vascular vasodilator capacity}

The sustained reactive hyperemia produced by ischemic exercise was measured in a semi-recumbent position, via venous occlusion plethysmography reported by Snell et al. (1987) with a minor modification. Briefly, the occlusion cuffs were wrapped around the left thigh and left ankle. Strain gauge was wrapped around the left calf at the heart level. After inflation of the ankle cuff at a pressure of $300 \mathrm{mmHg}$ to exclude foot blood flow, multiple baseline flow measurements were made at a thigh cuff pressure of $50 \mathrm{mmHg}$. Following the baseline measurements, the subject performed heel and toe-raising exercises for $2 \mathrm{~min}$ while the thigh cuff was inflated to $300 \mathrm{mmHg}$. After $1 \mathrm{~min}$ of recovery with the remaining thigh cuff inflation, the thigh cuff was deflated and multiple flow measurements were made at an occlusion cuff pressure of $50 \mathrm{mmHg}$. The measurements were repeated for $120 \mathrm{~s}$ with 4-s interval. Blood flow was calculated from the slope of the volume change over the first cardiac cycle. Peak calf vascular conductance (CVC) was calculated from peak flow divided by simultaneously measured MAP.

\section{STATISTICAL ANALYSES}

An unpaired t-test was used to determine the effects of habitual training on physiological characteristics and relative changes in variables of interest. Repeated-measures AVOVA by general linear models were performed to determine the effects of posture, acute exercise bouts, and regular exercise training on hemodynamic variables. In the case of a significant $F$-value, a Fischer's LSD posthoc test was used to identify significant differences among mean values. Simple correlation coefficients were calculated to determine the significant relation of interests. All data are reported as mean $\pm \mathrm{SD}$ or mean \pm s.e.m. Statistical significance was set a priori at $P<0.05$.

\section{RESULTS}

Selected physiological characteristics are summarized in Table $\mathbf{1 .}$ There were no significant group-differences in height, body weight, BMI, LVWT, LVM, LVM index, and FS. Endurancetrained men showed significantly larger LVEDD and lower LVWT index compared with the sedentary peers. Peak CVC was $63 \%$ higher in the endurance-trained than the sedentary peers $(P<0.0001)$

Target HR during main exercise (e.g., 75\% of HR reserve) and corresponding work rate were $136 \pm 4 \mathrm{bpm}$ and $94 \pm$ $14 \mathrm{~W}$ in the in the sedentary and $134 \pm 4 \mathrm{bpm}$ and $146 \pm$ $18 \mathrm{~W}$ in the endurance-trained groups, respectively. The exercise work rate was significantly higher in the endurance-trained than the sedentary group $(P<0.0001)$ although the target HR were similar between groups. All subjects completed the $1 \mathrm{~h}$ bout of exercise. Amount of ingested water was almost equivalent between the sedentary $(130 \pm 70 \mathrm{ml})$ and the endurancetrained $(130 \pm 50 \mathrm{ml})$. Following the exercise, body weight was significantly decreased in the sedentary by $0.55 \pm 0.20 \mathrm{~kg}$

Table 1 | Physiological characteristics.

\begin{tabular}{lcc}
\hline Variables & Sedentary & Endurance-trained \\
\hline Age (years) & $24 \pm 3$ & $22 \pm 5$ \\
Height (cm) & $174 \pm 6$ & $173 \pm 4$ \\
Body weight (kg) & $66.6 \pm 10.7$ & $61.4 \pm 3.6$ \\
BMI (kg/m²) & $22.2 \pm 2.5$ & $20.7 \pm 0.5$ \\
LVEDD (cm) & $4.4 \pm 0.4$ & $5.1 \pm 0.3^{*}$ \\
LVWT (cm) & $0.9 \pm 0.1$ & $0.8 \pm 0.1$ \\
LVWT/LVEDD (ratio) & $0.21 \pm 0.03$ & $0.16 \pm 0.03^{*}$ \\
LVM (g) & $151 \pm 37$ & $170 \pm 32$ \\
LVM index (g/m ${ }^{2}$ ) & $83 \pm 17$ & $97 \pm 17$ \\
Fractional shortening (\%) & $29.6 \pm 5.4$ & $31.8 \pm 5.8$ \\
Calf peak vascular & $0.35 \pm 0.1$ & $0.58 \pm 0.1^{*}$ \\
conductance (ml/min/100 & & \\
g/mmHg) & & \\
\hline
\end{tabular}

Data are mean and SD. BMI, body mass index; $L V E D D$, left ventricular enddiastolic diameter; LVWT, LV wall thickness; LVM, LV mass; LVM index, body surface-normalized LVM.

${ }^{*} P<0.05$ vs. sedentary. 
$(-0.8 \pm 0.3 \%)$ and in the endurance-trained by $1.17 \pm 0.21 \mathrm{~kg}$ $(-1.9 \pm 0.3 \%)(P<0.0001$ for both $)$. The extent of body weight loss was significantly larger in the endurance-trained $(P<$ 0.0001). Thus, post-exercise water ingestion was also greater in the endurance-trained $(351 \pm 63 \mathrm{ml})$ than the sedentary $(166 \pm 59 \mathrm{ml})(P<0.0001)$

\section{HRV AND BRS}

There were no significant group differences in both absolute and normalized values of HF power (Table 2). These values did not change significantly with exercise in both groups. HF power was significantly lowered during post-exercise HUT in the sedentary $(P<0.0001)$ but did not change in the endurancetrained either pre- or post-exercise HUT. Normalized HF power was significantly decreased with HUT in the sedentary both pre- and post-exercise ( $P=0.046$ and $P<0.0001$, respectively). In the endurance-trained, normalized HF power was significantly decreased with HUT pre-exercise $(P=0.004)$ but not post-exercise $(P=0.06)$.

Table 2 | Pre- and post-exercise heart rate variability and hemodynamic changes with 5-min head-up tilt (HUT).

\begin{tabular}{|c|c|c|c|c|c|}
\hline \multirow[b]{2}{*}{ Variables } & & \multicolumn{2}{|c|}{ Sedentary } & \multicolumn{2}{|c|}{ Endurance-trained } \\
\hline & & PRE & POST & PRE & POST \\
\hline HF power & Supine & $1112 \pm 963$ & $1629 \pm 3203$ & $853 \pm 870$ & $551 \pm 497$ \\
\hline$\left(\mathrm{ms}^{2}\right)$ & HUT & $350 \pm 263^{*}$ & $183 \pm 153^{*}$ & $247 \pm 250^{*}$ & $262 \pm 444$ \\
\hline Normalized HF & Supine & $0.23 \pm 0.15$ & $0.31 \pm 0.13$ & $0.23 \pm 0.10$ & $0.23 \pm 0.13$ \\
\hline power (a.u.) & HUT & $0.16 \pm 0.15^{*}$ & $0.12 \pm 0.07 *$ & $0.09 \pm 0.07 *$ & $0.15 \pm 0.16$ \\
\hline Systolic BP & Supine & $119 \pm 3$ & $116 \pm 3^{\dagger}$ & $115 \pm 3$ & $112 \pm 3$ \\
\hline$(\mathrm{mmHg})$ & HUT & $115 \pm 4^{*}$ & $110 \pm 4^{*}$ & $107 \pm 5^{*}$ & $102 \pm 3^{*}$ \\
\hline Diastolic BP & Supine & $66 \pm 2$ & $68 \pm 2$ & $62 \pm 2$ & $64 \pm 2$ \\
\hline$(\mathrm{mmHg})$ & HUT & $72 \pm 2^{*}$ & $73 \pm 3^{*}$ & $65 \pm 3^{*}$ & $66 \pm 3^{*}$ \\
\hline MAP & Supine & $85 \pm 2$ & $86 \pm 2$ & $82 \pm 3$ & $82 \pm 3$ \\
\hline$(\mathrm{mmHg})$ & HUT & $88 \pm 3$ & $87 \pm 3$ & $81 \pm 4$ & $79 \pm 3$ \\
\hline Heart rate & Supine & $56 \pm 3$ & $63 \pm 4^{\dagger}$ & $49 \pm 3^{\dagger}$ & $56 \pm 2^{\dagger}$ \\
\hline (beat/min) & HUT & $78 \pm 3^{*}$ & $91 \pm 5^{* \dagger}$ & $63 \pm 4^{* \ddagger}$ & $79 \pm 4^{*}$ \\
\hline SV & Supine & $103 \pm 4$ & $103 \pm 8$ & $118 \pm 8$ & $119 \pm 10$ \\
\hline$(\mathrm{ml})$ & HUT & $72 \pm 4^{*}$ & $71 \pm 5^{*}$ & $90 \pm 8^{*}$ & $85 \pm 9^{*}{ }^{\dagger}$ \\
\hline SV index & Supine & $57 \pm 3$ & $57 \pm 4$ & $67 \pm 4$ & $68 \pm 5$ \\
\hline$\left(\mathrm{ml} / \mathrm{m}^{2}\right)$ & HUT & $40 \pm 3^{*}$ & $39 \pm 3^{*}$ & $51 \pm 4^{*}$ & $49 \pm 5^{* \dagger}$ \\
\hline $\mathrm{CO}$ & Supine & $5.7 \pm 0.3$ & $6.4 \pm 0.3^{\dagger}$ & $5.8 \pm 0.5$ & $6.7 \pm 0.5^{\dagger}$ \\
\hline (L/min) & HUT & $5.6 \pm 0.4$ & $6.4 \pm 0.4^{\dagger}$ & $5.6 \pm 0.6$ & $6.6 \pm 0.7^{\dagger}$ \\
\hline CO index, & Supine & $3.2 \pm 0.1$ & $3.5 \pm 0.2^{\dagger}$ & $3.3 \pm 0.2$ & $3.8 \pm 0.3^{\dagger}$ \\
\hline$\left(\mathrm{L} / \mathrm{min} / \mathrm{m}^{2}\right)$ & HUT & $3.1 \pm 0.2$ & $3.6 \pm 0.3^{\dagger}$ & $3.2 \pm 0.3$ & $3.8 \pm 0.3^{\dagger}$ \\
\hline TPR & Supine & $15.2 \pm 0.8$ & $13.8 \pm 0.8^{\dagger}$ & $15.0 \pm 1.5$ & $12.7 \pm 0.7^{\dagger}$ \\
\hline (a.u.) & HUT & $16.3 \pm 1.3$ & $14.2 \pm 1.2^{\dagger}$ & $15.7 \pm 2.0$ & $12.6 \pm 1.0^{\dagger}$ \\
\hline TPR index & Supine & $8.5 \pm 0.5$ & $7.7 \pm 0.5^{\dagger}$ & $8.6 \pm 1.0$ & $7.3 \pm 0.5^{\dagger}$ \\
\hline (a.u.) & HUT & $9.1 \pm 0.8$ & $7.9 \pm 0.7^{\dagger}$ & $9.1 \pm 1.3$ & $7.2 \pm 0.7^{\dagger}$ \\
\hline
\end{tabular}

Data are mean $\pm S D$. $H F$, high frequency spectral power of $R$ - $R$ interval; $B P$, blood pressure; MAP, mean arterial pressure; HR, heart rate; SV, stroke volume; CO, cardiac output; TPR, total peripheral resistance. SV index, CO index, and TPR index indicate body surface area-normalized values.

${ }^{*} P<0.05$ vs. supine position.

${ }^{\dagger} P<0.05$ vs. pre-exercise.

${ }^{\ddagger} P<0.05$ vs. sedentary.
The individual changes in $\triangle \mathrm{HR} / \triangle \mathrm{MAP}$ were presented in Figure 2. $\triangle \mathrm{HR} / \triangle \mathrm{MAP}$ tended to be lower in the endurancetrained compared with their sedentary peers $(P=0.05)$. It was augmented after the exercise bout in the endurance-trained $(P=$ $0.007)$ but not in the sedentary peers $(P=0.07)$.

\section{HEMODYNAMIC VARIABLES}

\section{Supine rest}

After the exercise, $\mathrm{HR}$ and $\mathrm{CO}$ were significantly higher $(P<0.0001$, vs. pre-exercise), and TPR was significantly lower $(P<0.0001$, vs. pre-exercise) in both groups (Table 2). SV, DBP, and MAP remained unchanged after the exercise in both groups. SBP was significantly lowered in the sedentary $(P=0.03$, vs. pre-exercise) and tended to be lowered in the endurance-trained ( $P=0.08$, vs. pre-exercise) after the exercise bout.

\section{HEMODYNAMIC RESPONSES TO SUSTAINED HUT}

During sustained HUT, HR, CO, and CO index were significantly higher after the exercise bout in both groups $(P<0.001$, vs. preexercise) (Table 2). The SV and SV index remained unchanged in the sedentary but were significantly decreased in the endurancetrained after the exercise bout $(P=0.02$, vs. pre-exercise). The TPR and TPR index were significantly lower after the exercise bout in both groups $(P<0.0001$, vs. pre-exercise). SBP was significantly lower $(P<0.0001$, vs. pre-exercise $)$ but DBP and MAP remained unchanged after the exercise bout in both groups.

As shown in Figure 3, the decline in SV during the sustained HUT was significantly augmented after the exercise bout in the endurance-trained $(P=0.03)$ but not in the sedentary. The increases in HR during the sustained HUT were significantly augmented following the exercise bout in both groups (sedentary: $P=0.02$; Endurance-trained: $P=0.002$ ). These responses were not affected by the elevated baseline (supine) $\mathrm{HR}$ at the start of post-exercise HUT $(r=0.146, P=0.58)$. The augmented response of HUT-induced tachycardia was greater in the endurance-trained than in their sedentary peers (2.0-fold vs. 1.3 -fold, $P=0.043$, Figure 4$)$. In pooled subjects, post-exercise change in sustained HUT-induced tachycardia correlated with corresponding change in HUT-induced SV response $(r=0.494$, $P=0.044$, Figure 5).

\section{DISCUSSION}

Main findings of this study were as follows: In addition to the reduced TPR, HUT-induced decline in SV, a risk for orthostatic intolerance, was significantly augmented after the exercise in the endurance-trained. Nevertheless, orthostatic MAP was well maintained. Tachycardia response during sustained orthostasis was doubled after the exercise. Additionally, cardiovagal BRS was improved after the exercise in the endurance-trained but not in the sedentary. These results suggest that in the endurance-trained men, the increases in orthostatic tachycardia and cardiovagal BRS may favorably mitigate accumulated risks for orthostatic intolerance in the early phase of post-exercise.

Hemodynamic regulation via arterial baroreflex to orthostatic stress is an important protective mechanism to regulate arterial BP and cerebral blood flow (Van Lieshout et al., 2003). In healthy subjects without autonomic failure or cardiac 

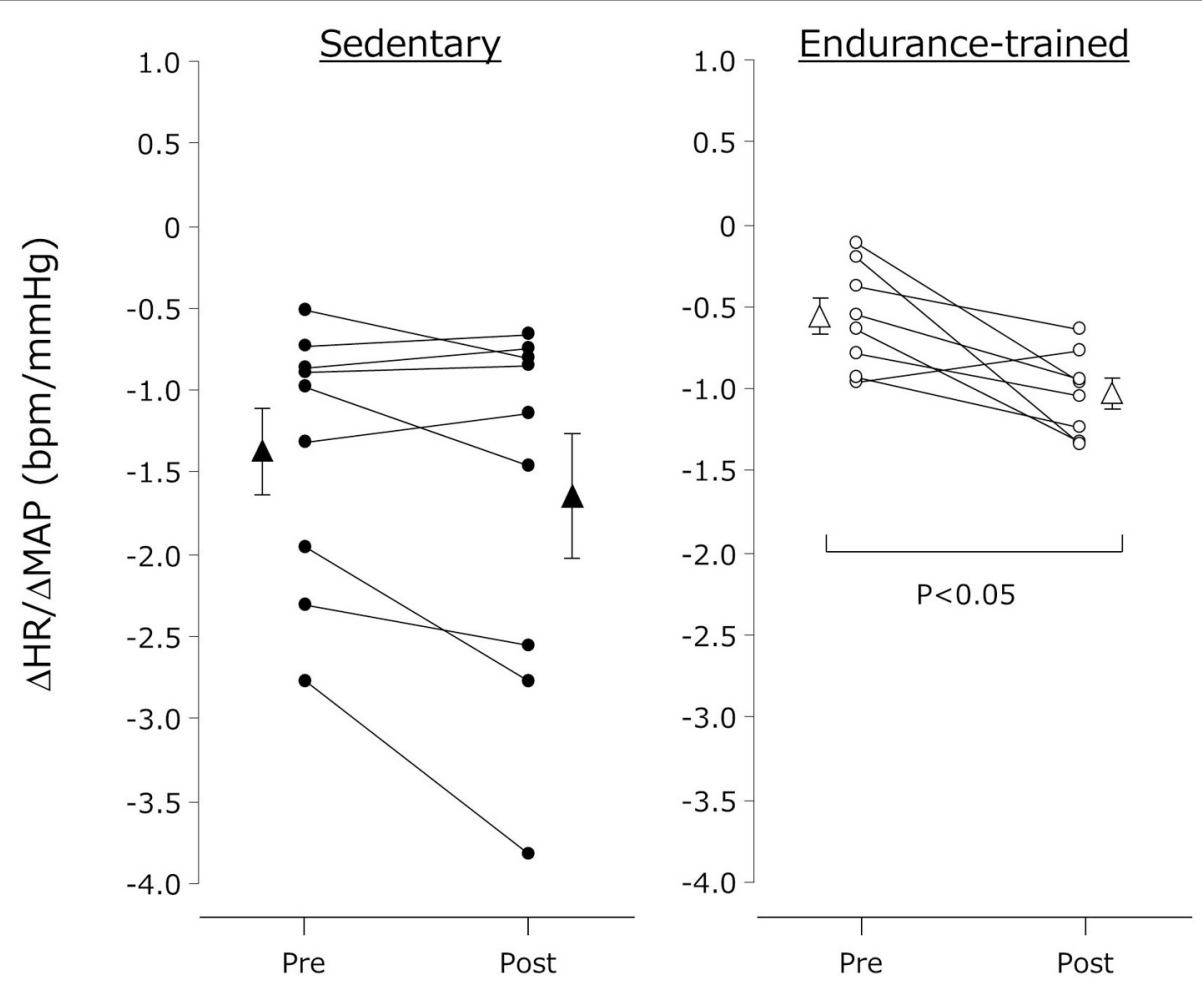

FIGURE 2 | Cardiovagal baroreflex sensitivity before and after the dynamic exercise bout. Cardiovagal baroreflex sensitivity was quantified the ratio of HUT-induced reduction in MAP (from supine MAP to the nadir MAP observed within several seconds after the HUT stimulation) to the increase in
HR (from supine HR to the peak HR after the HUT stimulation) (e.g., $\triangle \mathrm{HR} / \triangle \mathrm{MAP}$. Closed (sedentary) and open (endurance-trained) circles indicate individual data. Closed (sedentary) and open (endurance-trained) triangles indicate mean \pm s.e. $m$ dysfunction the arterial baroreflexes correct an abrupt drop of BP induced by orthostatic stress through sympathetic nerve activation via tachycardia and vasoconstriction, respectively (Cooper and Hainsworth, 2001, 2002; Ogoh et al., 2003; Arbab-Zadeh et al., 2004; Fu et al., 2004). However, endurance-trained individuals are predisposed to orthostatic hypotension or intolerance (Convertino, 1993). This could be attributed to higher leg maximal vasodilator capacity, greater decline in SV during orthostatic stimulation, and lower BRS (Levine et al., 1991; Ogoh et al., 2003).

As expected, in the present study, the endurance-trained exhibited significantly higher leg vasodilator capacity compared with the sedentary. Furthermore, in addition to the reduction in TPR during post-exercise HUT, a greater augmentation of HUTinduced SV drop was identified in the endurance-trained men. Nevertheless, MAP was well maintained during post-exercise HUT. These phenomena imply the presence of a compensatory mechanism responsible for the favorable BP regulation during post-exercise orthostatic stimulation. In this context, we can speculate possible mechanisms. First, endurance athletes exhibited a greater augmentation of HUT-induced tachycardia following the exercise bout compared with their sedentary peers (2.0-fold vs. 1.3 -fold, $P=0.04)$. Importantly, the change in orthostatic tachycardia response with the exercise bout significantly correlated with the corresponding change in HUT-induced SV fall, as shown in Figure 5. These results suggest that the increased tachycardia response might compensate the substantial reduction of SV during the sustained orthostasis after the exercise bout. Indeed, $\mathrm{CO}$ was increased rather than decreased during orthostasis after the exercise despite a large reduction in SV. Second, cardiovagal BRS was significantly improved after the exercise in the endurance-trained. Our findings were consistent with previous reports that baroreflex HR gain was augmented after moderateintensity dynamic exercise, as assessed using nonpharmacological (Halliwill et al., 1996b) or pharmacological (Somers et al., 1985) techniques. Taken together, in endurance-trained individuals, post-exercise augmentation of orthostatic tachycardia as well as increased cardiovagal BRS may favorably mitigate accumulated risks for orthostatic intolerance in the early phase of post-exercise. In this study, the absolute workload of exercise differed between 2 groups because each subject underwent the exercise at the relatively same intensity (e.g., 75\% HR reserve). Whether the differences of workload and weight loss were associated with the different responses of post-exercise orthostatic tachycardia and/or BRS change would be warranted by future studies. Further investigations are also needed in different subjects (i.e., women, fainters) and exercise conditions (i.e., prolonged exercise).

It is well-known that $\mathrm{BP}$ reduces following a single bout of dynamic exercise in most individuals, which is called post-exercise hypotension (Kenney and Seals, 1993; Halliwill, 2001). Post-exercise hypotension in healthy sedentary people is described as a sustained increase in systemic vascular conductance which is not completely offset by ongoing elevations in $\mathrm{CO}$ 

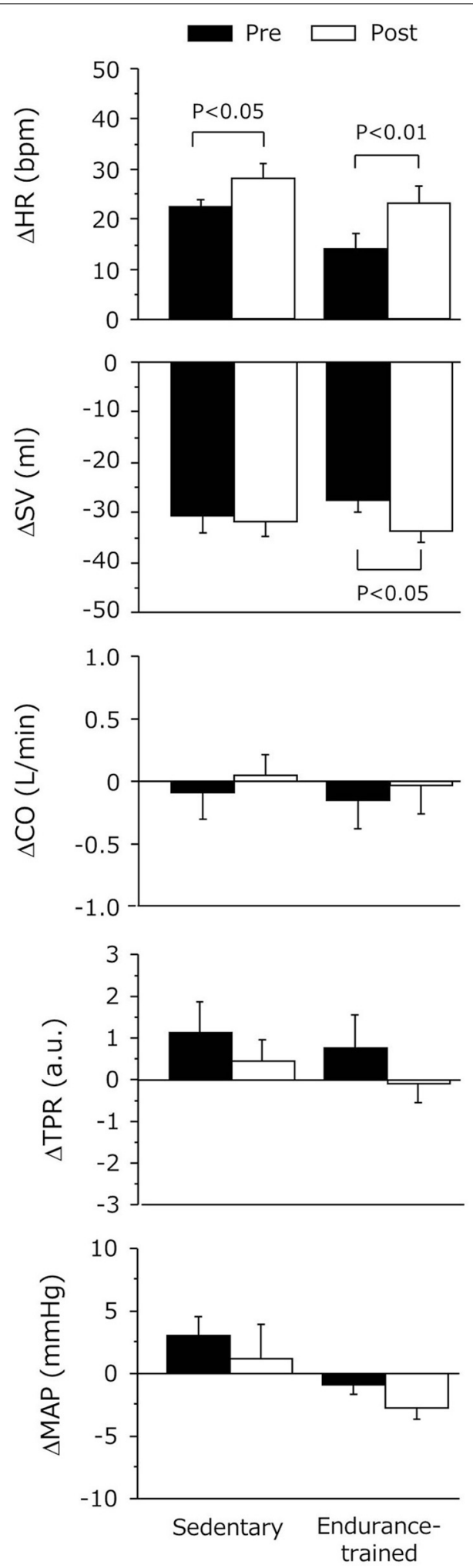

FIGURE 3 | Hemodynamic changes during 5-min orthostatic stimulation before and after the dynamic exercise bout. Data are mean and s.e.m. SV, stroke volume; HR, heart rate; $C O$, cardiac output; TPR, total peripheral resistance; MAP, mean arterial pressure. Delta $(\Delta)$ indicates the difference from the baseline to sustained HUT.

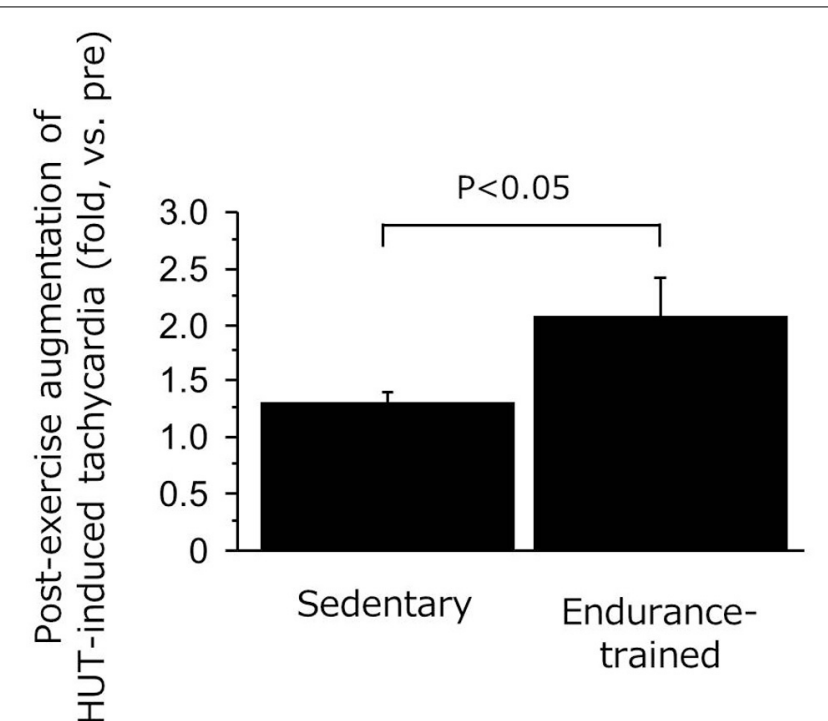

FIGURE 4 | Ratio of HUT-induced tachycardia responses (post/pre) in the sedentary and endurance-trained men. HUT-induced tachycardia response was calculated as the heart rate during HUT minus heart rate in supine position. Data are mean \pm s.e.m.

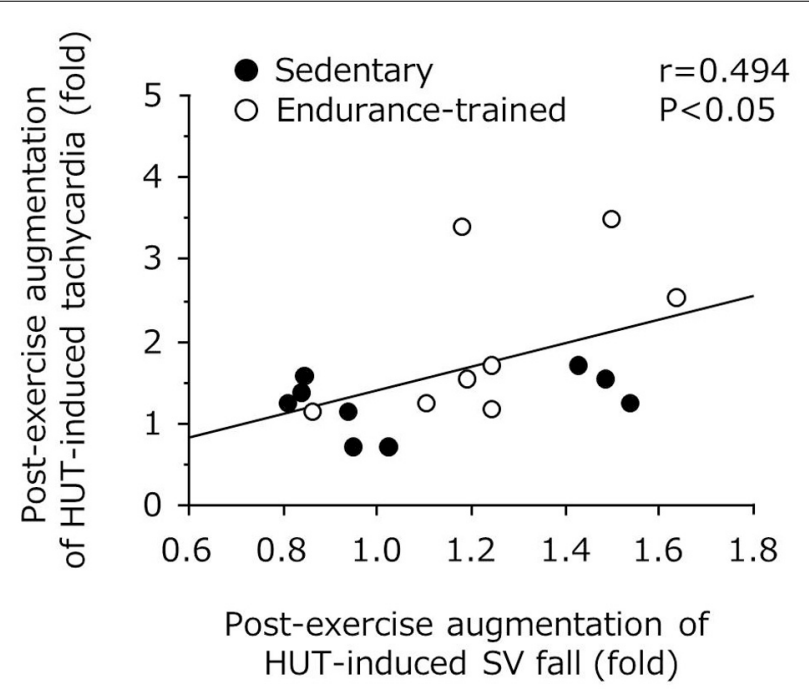

FIGURE 5 | Correlation between post-exercise augmentations of stroke volume (SV) fall and tachycardia with $5-\mathrm{min}$ orthostatic stimulation.

(Halliwill, 2001), whereas in endurance-trained men it may be attributed to a reduction in CO rather than the decreased TPR (Senitko et al., 2002; Dujic et al., 2006). In this study, the postexercise reductions in supine MAP were not manifest in both groups. Such a discrepancy might be explained by the differences in the experimental protocol, including fluid intake. Lynn et al. (Lynn et al., 2009) suggested that water intake mitigated the post-exercise reduction in CO. In this study, subjects drank water corresponding to $30 \%$ of sweat loss for prevention of dehydration and to control the possible influence of water ingestion 
on hemodynamics (Scott et al., 2001). Although the reason for the absence of post-exercise hypotension remains unknown, it might be due to the post-exercise "increase" in CO observed in the current study. The sustained increase in CO after the exercise might be due to the increase in cutaneous blood flow for thermoregulation.

Several experimental considerations are acknowledged. First, we did not measure aerobic capacity (i.e., maximal oxygen uptake). However, we identified chronic endurance trainingspecific vascular adaptation, such as higher calf vasodilator capacity in the endurance-trained, suggesting that the exercise training regimes of our trained individuals were sufficient to accomplish our aim. Second, the exercise intensity was set by HR reserve calculated from the age-predicted maximal HR. Third, we applied only hypotensive perturbation to evaluate cardiovagal BRS. Use of combined hypotensive and hypertensive perturbation could provide more detailed information about BRS (Ogoh et al., 2003). Forth, there may be small differences between beat-tobeat SV estimated by the Modelflow method and that measured by Doppler echocardiography, an established reliable technique (Sugawara et al., 2003). In order to minimize the Modelflowderived estimation error, we calibrated it using Doppler echocardiography method. BP we reported (e.g., brachium level) was also estimated by a filtering of the finger arterial waveforms. Finally, as we compared post-exercise hemodynamic regulation between groups by cross-sectional study design, genetic influences could not be completely ruled out.

In conclusion, we determined the regular endurance trainingspecific adaptation of post-exercise hemodynamic regulation during orthostatic stress. Orthostatic MAP was well maintained in the endurance athletes despite the augmentation of HUT-induced decline in SV after the exercise. These results might be partly explained by the post-exercise increase in tachycardia response during orthostasis. Additionally, cardiovagal BRS was enhanced after the exercise in the endurance-trained but not in the sedentary. Taken together, the increases in orthostatic tachycardia and cardiovagal BRS may favorably mitigate accumulated risks for orthostatic intolerance in the early phase of post-exercise in the endurance athletes. The impact of the impaired compensatory mechanisms on orthostatic intolerance, such as the case when $\mathrm{HR}$ is already elevated and can no longer increase with orthostatic stimulation (i.e., ceiling effect), should be investigated by a future study.

\section{ACKNOWLEDGMENTS}

This study was supported by The Nakatomi Foundation (Jun Sugawara) and The Uehara Memorial Foundation (Jun Sugawara). The authors thank Michael Schulman for his technical assistance.

\section{REFERENCES}

Arbab-Zadeh, A., Dijk, E., Prasad, A., Fu, Q., Torres, P., Zhang, R., et al. (2004). Effect of aging and physical activity on left ventricular compliance. Circulation 110, 1799-1805. doi: 10.1161/01.CIR.0000142863.71285.74

Bhagyalakshmi, A., and Frangos, J. A. (1989). Mechanism of shear-induced prostacyclin production in endothelial cells. Biochem. Biophys. Res. Commun. 158, 31-37. doi: 10.1016/S0006-291X(89)80172-X

Cheitlin, M. D., Armstrong, W. F., Aurigemma, G. P., Beller, G. A., Bierman, F. Z., Davis, J. L., et al. (2003). ACC/AHA/ASE 2003 guideline update for the clinical application of echocardiography: summary article: a report of the American College of Cardiology/American Heart Association Task Force on Practice Guidelines (ACC/AHA/ASE Committee to Update the 1997 Guidelines for the Clinical Application of Echocardiography). Circulation 108, 1146-1162. doi: 10.1161/01.CIR.0000073597.57414.A9

Convertino, V. A. (1993). Endurance exercise training: conditions of enhanced hemodynamic responses and tolerance to LBNP. Med. Sci. Sports Exerc. 25, 705-712. doi: 10.1249/00005768-199306000-00010

Cooper, V. L., and Hainsworth, R. (2001). Carotid baroreceptor reflexes in humans during orthostatic stress. Exp. Physiol. 86, 677-681. doi: 10.1113/eph8602213

Cooper, V. L., and Hainsworth, R. (2002). Effects of head-up tilting on baroreceptor control in subjects with different tolerances to orthostatic stress. Clin. Sci. (Lond.) 103, 221-226.

Devereux, R. B., and Reichek, N. (1977). Echocardiographic determination of left ventricular mass in man. Anatomic validation of the method. Circulation 55, 613-618. doi: 10.1161/01.CIR.55.4.613

Dinenno, F. A., and Joyner, M. J. (2004). Combined NO and PG inhibition augments alpha-adrenergic vasoconstriction in contracting human skeletal muscle. Am. J. Physiol. Heart Circ. Physiol. 287, H2576-H2584. doi: 10.1152/ajpheart.00621.2004

Dujic, Z., Ivancev, V., Valic, Z., Bakovic, D., Marinovic-Terzic, I., Eterovic, D., et al. (2006). Postexercise hypotension in moderately trained athletes after maximal exercise. Med. Sci. Sports Exerc. 38, 318-322. doi: 10.1249/01.mss.0000187460.73235.3b

Emhoff, C. A., Barrett-O'Keefe, Z., Padgett, R. C., Hawn, J. A., and Halliwill, J. R. (2011). Histamine-receptor blockade reduces blood flow but not muscle glucose uptake during postexercise recovery in humans. Exp. Physiol. 96, 664-673. doi: 10.1113/expphysiol.2010.056150

Fu, Q., Witkowski, S., and Levine, B. D. (2004). Vasoconstrictor reserve and sympathetic neural control of orthostasis. Circulation 110, 2931-2937. doi: 10.1161/01.CIR.0000146384.91715.B5

Halliwill, J. R. (2001). Mechanisms and clinical implications of post-exercise hypotension in humans. Exerc. Sport Sci. Rev. 29, 65-70. doi: 10.1097/00003677200104000-00005

Halliwill, J. R., Taylor, J. A., and Eckberg, D. L. (1996a). Impaired sympathetic vascular regulation in humans after acute dynamic exercise. J Physiol 495(Pt 1), 279-288.

Halliwill, J. R., Taylor, J. A., Hartwig, T. D., and Eckberg, D. L. (1996b). Augmented baroreflex heart rate gain after moderate-intensity, dynamic exercise. Am. J. Physiol. 270, R420-R426.

Joyner, M. J., and Casey, D. P. (2014). Muscle blood flow, hypoxia, and hypoperfusion. J. Appl. Physiol. (1985) 116, 852-857. doi: 10.1152/japplphysiol.006 20.2013

Kenney, M. J., and Seals, D. R. (1993). Postexercise hypotension. Key features, mechanisms, and clinical significance. Hypertension 22, 653-664. doi: 10.1161/01.HYP.22.5.653

Levine, B. D., Lane, L. D., Buckey, J. C., Friedman, D. B., and Blomqvist, C. G. (1991). Left ventricular pressure-volume and Frank-Starling relations in endurance athletes. Implications for orthostatic tolerance and exercise performance. Circulation 84, 1016-1023. doi: 10.1161/01.CIR.84.3.1016

Lucas, S. J., Cotter, J. D., Murrell, C., Wilson, L., Anson, J. G., Gaze, D., et al. (2008). Mechanisms of orthostatic intolerance following very prolonged exercise. J. Appl. Physiol. 105, 213-225. doi: 10.1152/japplphysiol.001 75.2008

Lynn, B. M., Minson, C. T., and Halliwill, J. R. (2009). Fluid replacement and heat stress during exercise alter post-exercise cardiac haemodynamics in endurance exercise-trained men. J. Physiol. (Lond.) 587, 3605-3617. doi: 10.1113/jphysiol.2009.171199

Murrell, C. J., Cotter, J. D., George, K., Shave, R., Wilson, L., Thomas, K., et al. (2011). Syncope is unrelated to supine and postural hypotension following prolonged exercise. Eur. J. Appl. Physiol. 111, 469-476. doi: 10.1007/s00421-0101671-8

Nagao, T., and Vanhoutte, P. M. (1993). Endothelium-derived hyperpolarizing factor and endothelium-dependent relaxations. Am. J. Respir. Cell Mol. Biol. 8, 1-6. doi: 10.1165/ajrcmb/8.1.1

Ogoh, S., Volianitis, S., Nissen, P., Wray, D. W., Secher, N. H., and Raven, P. B. (2003). Carotid baroreflex responsiveness to head-up tilt-induced central hypovolaemia: effect of aerobic fitness. J. Physiol. 551, 601-608. doi: 10.1113/jphysiol.2003.046029 
Scott, E. M., Greenwood, J. P., Gilbey, S. G., Stoker, J. B., and Mary, D. A. (2001). Water ingestion increases sympathetic vasoconstrictor discharge in normal human subjects. Clin. Sci. (Lond.) 100, 335-342. doi: 10.1042/CS20000177

Senitko, A. N., Charkoudian, N., and Halliwill, J. R. (2002). Influence of endurance exercise training status and gender on postexercise hypotension. J Appl Physiol 92, 2368-2374. doi: 10.1152/japplphysiol.00020.2002

Smith, M. L., and Raven, P. B. (1986). Cardiovascular responses to lower body negative pressure in endurance and static exercise-trained men. Med. Sci. Sports Exerc. 18, 545-550. doi: 10.1249/00005768-198610000-00009

Snell, P. G., Martin, W. H., Buckey, J. C., and Blomqvist, C. G. (1987). Maximal vascular leg conductance in trained and untrained men. J. Appl. Physiol. (1985) $62,606-610$.

Somers, V. K., Conway, J., Lewinter, M., and Sleight, P. (1985). The role of baroreflex sensitivity in post-exercise hypotension. J. Hypertens. 3, S129-S130.

Sugawara, J., Tanabe, T., Miyachi, M., Yamamoto, K., Takahashi, K., Iemitsu, M., et al. (2003). Non-invasive assessment of cardiac output during exercise in healthy young humans: comparison between Modelflow method and Doppler echocardiography method. Acta Physiol. Scand. 179, 361-366. doi: 10.1046/j.0001-6772.2003.01211.x

Task Force of the European Society of Cardiology and the North American Society of Pacing and Electrophysiology. (1996). Heart rate variability: standards of measurement, physiological interpretation and clinical use. Circulation 93, 1043-1065. doi: 10.1161/01.CIR.93.5.1043

Van Lieshout, J. J., Wieling, W., Karemaker, J. M., and Secher, N. H. (2003). Syncope, cerebral perfusion, and oxygenation. J. Appl. Physiol. 94, 833-848. doi: 10.1152/japplphysiol.00260.2002
Victor, R. G., and Leimbach, W. N. Jr. (1987). Effects of lower body negative pressure on sympathetic discharge to leg muscles in humans. J. Appl. Physiol. 63, 2558-2562.

Wesseling, K. H., Jansen, J. R., Settels, J. J., and Schreuder, J. J. (1993). Computation of aortic flow from pressure in humans using a nonlinear, three-element model. J. Appl. Physiol. 74, 2566-2573.

Willie, C. K., Ainslie, P. N., Taylor, C. E., Eves, N. D., and Tzeng, Y. C. (2013). Maintained cerebrovascular function during post-exercise hypotension. Eur. J. Appl. Physiol. 113, 1597-1604. doi: 10.1007/s00421-012-2578-3

Conflict of Interest Statement: The authors declare that the research was conducted in the absence of any commercial or financial relationships that could be construed as a potential conflict of interest.

Received: 12 February 2014; accepted: 02 June 2014; published online: 24 June 2014. Citation: Sugawara J, Komine H, Miyazawa T, Imai T and Ogoh S (2014) Influence of regular exercise training on post-exercise hemodynamic regulation to orthostatic challenge. Front. Physiol. 5:229. doi: 10.3389/fphys.2014.00229

This article was submitted to Integrative Physiology, a section of the journal Frontiers in Physiology.

Copyright (c) 2014 Sugawara, Komine, Miyazawa, Imai and Ogoh. This is an openaccess article distributed under the terms of the Creative Commons Attribution License (CC BY). The use, distribution or reproduction in other forums is permitted, provided the original author(s) or licensor are credited and that the original publication in this journal is cited, in accordance with accepted academic practice. No use, distribution or reproduction is permitted which does not comply with these terms. 